\title{
O Fazer Emergir na Ação Educativa: Compreensões Sobre o Operar das Tecnologias Digitais no Ensinar e no Aprender Matemática em uma Cultura Digital na Universidade
}

\author{
El Hacer emerger en la Acción Educativa: Comprensiones Sobre el \\ Operar de las Tecnologías Digitales en la Enseñanza y Aprender \\ Matemáticas en una Cultura Digital en la Universidad \\ The Doing Emerge in Educational Action: Comprehensions on the \\ Operation of Digital Technologies in Teaching and Learning Mathematics \\ in a Digital Culture in the University
}

\author{
Daniel da Silva Silveira ${ }^{1}$ \\ Daniele Amaral Fonseca ${ }^{2}$ \\ Gerson Freitas $\mathrm{Luz}^{3}$ \\ Débora Pereira Laurino 4
}

\begin{abstract}
Resumo
Este trabalho é um recorte de uma pesquisa de doutorado a qual objetivou compreender a discursividade em relação ao operar das tecnologias digitais de um coletivo de professores que constituiu uma rede de conversação em um grupo de pesquisa na área de educação a distância e tecnologias de uma Universidade Federal. A pesquisa é balizada pelo caminho explicativo da objetividade entre parênteses na qual os pesquisadores são observadores implicados e foi utilizado a técnica do Discurso do Sujeito Coletivo para analisar os registros produzidos em fóruns. Nesse artigo é analisado e problematizado o discurso coletivo intitulado "O aprender matemática enatuado na docência pelas tecnologias digitais" o qual evidenciou que diferentes experiências de ação e reflexão sobre o operar das tecnologias digitais instigam o estudante a experimentar, fazer e interagir, o que permite significar e aprender a Matemática em uma cultura digital. Assim, com este trabalho conclui-se que a incorporação das tecnologias digitais viabilizando a expressão, o compartilhamento e a produção de ações, emoções e experiências em um coordenar recorrente e recursivo poderá viabilizar uma proposta de formação no espaço universitário que ultrapasse a sala de aula, que potencialize o acesso e a convivência em uma cultura digital trazendo desafios à prática pedagógica.
\end{abstract}

Palavras-Chave: Cultura Digital; Professores de Matemática; Rede de Conversação.

\section{Resumen}

Este trabajo es un recorte de una investigación de doctorado la cual objetivó comprender la discursividad en relación al operar de las tecnologías digitales de un colectivo de profesores que constituyó una red de conversación en un grupo de investigación en el área de educación a distancia y tecnologías de una Universidad federal. La investigación es balizada por el camino explicativo de la objetividad entre paréntesis en la cual los

\footnotetext{
${ }^{1}$ Doutor em Educação em Ciências; Universidade Federal do Rio Grande - FURG; Rio Grande, Rio Grande do Sul, Brasil; dssilveira@furg.br

${ }^{2}$ Licenciada em Matemática; Universidade Federal do Rio Grande - FURG; Rio Grande, Rio Grande do Sul, Brasil; danieleamaral4@gmail.com

${ }^{3}$ Acadêmico do Curso de Licenciatura em Matemática; Universidade Federal do Rio Grande - FURG; Rio Grande, Rio Grande do Sul, Brasil; gersonluz2000@gmail.com

${ }^{4}$ Doutora em Informática na Educação; Universidade Federal do Rio Grande - FURG; Rio Grande, Rio Grande do Sul, Brasil; deboralaurino@furg.br
} 
investigadores son observadores implicados y se utilizó la técnica del Discurso del Sujeto Colectivo para analizar los registros producidos en foros. En este artículo se analiza y cuestiona el discurso colectivo titulado "El aprendizaje matemático en la docencia por las tecnologías digitales" que evidenció que diferentes experiencias de acción y reflexión sobre el funcionamiento de las tecnologías digitales instigan al estudiante a experimentar, hacer e interactuar, lo que permite significar y aprender matemáticas en una cultura digital. Así, con este trabajo se concluye que la incorporación de las tecnologías digitales viabilizando la expresión, el compartir y la producción de acciones, emociones y experiencias en un coordinar recurrente y recursivo podrá viabilizar una propuesta de formación en el espacio universitario que sobrepase el aula, que potencie el acceso y la convivencia en una cultura digital trayendo desafíos a la práctica pedagógica.

Palabras claves: Cultura Digital; Profesores de Matemáticas; Red de Conversación.

\begin{abstract}
This work is a cut of a doctoral research that aimed to understand the discursiveness in relation to the operation of the digital technologies of a collective of professors that constituted a network of conversation in a group of research in the area of distance education and technologies of a University Federal. The research is based on the explanatory path of parenthetical objectivity in which researchers are involved observers and the Collective Subject Discourse technique was used to analyze the records produced in forums. In this article is analyzed and problematized the collective discourse entitled "The mathematical learning enactioned in teaching by digital technologies" which showed that different experiences of action and reflection on the operation of digital technologies instigate the student to experiment, to do and to interact, which allows signify and learn Mathematics in a digital culture. Therefore, it is possible to conclude with this work that the incorporation of digital technologies enabling the expression, sharing and production of actions, emotions and experiences in a recurrent and recursive co-ordination can make possible a proposal of formation in the university space that surpasses the classroom, which enhances access and coexistence in a digital culture bringing challenges to pedagogical practice.
\end{abstract}

Keywords: Digital Culture; Mathematics Teachers; Network Conversation.

\title{
1. Introdução
}

Refletir sobre as influências que a globalização econômica, política e social têm no cotidiano das pessoas implica em discorrer sobre a importância que as tecnologias digitais exercem na educação e na forma de fazer ciência. O campo educacional, como parte do sistema social, é influenciado e influencia a produção do conhecimento a partir do operar das tecnologias digitais, o que pode transformar e/ou ressignificar os modos de pensar a formação universitária dos sujeitos.

Uma cultura envolve os modos de viver, os sistemas de valores e crenças, os instrumentos de trabalho, os tipos de organização social, político e econômico, bem como os modos de pensar e agir dos sujeitos. Para Pretto (2011) diferentes fenômenos caóticos e relações não-lineares contribuíram para as transformações da contemporaneidade e ampliaram as relações entre tecnologia e cultura.

Uma cultura surge a partir de uma dinâmica sistêmica, na qual a rede de conversação de uma comunidade vive configurada pelo emocionar, que começa a se conservar no aprender dos sujeitos. Assim, o modo como se vive é o que fundamenta o mecanismo de conservação da cultura vivida (MATURANA e VERDEN-ZÖLLER, 2004). Qualquer mudança estrutural 
que ocorra por meio de coordenações de ações gera novos fluxos dinâmicos de interações em uma rede de conversação.

Nesse fluxo, as mudanças estruturais podem acontecer quando a estrutura do sujeito estabelece uma relação mútua e dinâmica com o contexto de suas práticas imbricados as tecnologias digitais, possibilitando que aconteça um domínio de estados e de perturbações na estrutura do sujeito. Assim, as operações que provocam mudanças estruturais como os modos de agir, pensar, interagir e conviver resultam em um aprender.

Sabe-se que os estudantes serão confrontados com situações que exigirão diferentes conhecimentos e mecanismos para resolvê-las. Sendo assim, pensar em mudanças curriculares, tais como, aproximar os conteúdos visto em sala de aula do contexto desses sujeitos e criar atividades pedagógicas pautadas em novas dinâmicas de interação que incluam as tecnologias digitais no fazer humano, é uma possibilidade para o desenvolvimento cognitivo, afetivo e social dos estudantes.

Desta forma, o objetivo deste trabalho é compreender a discursividade em relação ao operar das tecnologias digitais de um coletivo de professores que constituiu uma rede de conversação em um grupo de pesquisa na área de educação a distância e tecnologias de uma Universidade Federal. Para compreender a discursividade desta rede de conversação a pesquisa foi balizada pelo caminho explicativo da objetividade entre parênteses e utilizou-se a técnica do Discurso do Sujeito Coletivo (DSC) para gerar o discurso e analisá-lo imbricado as concepções de autores que discutem acerca das tecnologias digitais no campo educacional. Este procedimento metodológico será apresentado na próxima seção.

\section{Processo metodológico}

O presente trabalho é um recorte de uma investigação de doutoramento realizada com integrantes do Grupo de Pesquisa Educação a Distância e Tecnologias (EaD-TEC). Fazem parte deste grupo atualmente 20 doutores e um mestre, todos docentes de Universidades Públicas Federais com uma média de 15 anos de docência, além de estudantes de doutorado, mestrado e alunos de graduação. O grupo realiza seus estudos sobre o imbricamento das tecnologias digitais e das metodologias em ambientes educativos sustentados por uma Ecologia Cognitiva Digital (relações e interações que se estabelecem na rede entre os sujeitos e o meio onde vivem, com suas recíprocas influências).

Apresenta-se, na Tabela 1, o perfil dos integrantes pesquisados a partir da sua formação acadêmica, o ano de conclusão na formação inicial e o ano que ingressaram no grupo de pesquisa. Cabe salientar que nem todos os integrantes do grupo de pesquisa atuam 
RELACult - Revista Latino-Americana de Estudos em Cultura e Sociedade

Revista Latinoamericana de Estudios en Cultura y Sociedad | Latin American Journal of Studies in Culture and Society V. 05, ed. especial, abr., 2019, artigo n ${ }^{\circ} 1189$ | claec.org/relacult |e-ISSN: 2525-7870

no Ensino Superior, no entanto, se aproximam da pesquisa pelo fato de estudarem e operarem as tecnologias digitais no contexto profissional ou acadêmico.

Tabela 1 - Perfil dos sujeitos que compõem a pesquisa.

\begin{tabular}{|c|c|c|c|}
\hline Integrante & Formação & $\begin{array}{l}\text { Ano de } \\
\text { conclusão da } \\
\text { formação inicial }\end{array}$ & $\begin{array}{l}\text { Ingresso no } \\
\text { Grupo de } \\
\text { Pesquisa }\end{array}$ \\
\hline 1 & $\begin{array}{l}\text { Licenciada em Matemática, especialista em Tecnologias da } \\
\text { Informação e Comunicação, mestre em Engenharia } \\
\text { Oceânica, cursando doutorado em Educação em Ciências }\end{array}$ & 1997 & 2013 \\
\hline 2 & $\begin{array}{l}\text { Licenciada em Matemática, cursando mestrado em } \\
\text { Educação em Ciências }\end{array}$ & 2015 & 2014 \\
\hline 3 & $\begin{array}{c}\text { Bacharelado em Engenharia Elétrica, especialista em } \\
\text { Ciência da Computação, mestre em Computação, cursando } \\
\text { doutorado em Educação em Ciências }\end{array}$ & 1995 & 2012 \\
\hline 4 & $\begin{array}{l}\text { Licenciado em Matemática, mestre em Educação em } \\
\text { Ciências, cursando doutorado em Educação em Ciências. }\end{array}$ & 2008 & 2007 \\
\hline 5 & $\begin{array}{l}\text { Licenciada em Matemática, especialista em Ciências da } \\
\text { Computação, mestre em Computação, doutora em } \\
\text { Informática na Educação }\end{array}$ & 1990 & 2002 \\
\hline 6 & $\begin{array}{l}\text { Licenciada em Matemática, especialista em Metodologia } \\
\text { do Ensino de Matemática e Física, mestre em Ensino de } \\
\text { Ciências e Matemática, cursando doutorado em Educação } \\
\text { em Ciências }\end{array}$ & 2004 & 2016 \\
\hline 7 & $\begin{array}{c}\text { Licenciada em Letras - Português, cursando Especialização } \\
\text { em Tecnologias da Informação e Comunicação na } \\
\text { Educação }\end{array}$ & 2010 & 2016 \\
\hline 8 & $\begin{array}{l}\text { Licenciada em Matemática, especialista em Tecnologias da } \\
\text { Informação e Comunicação, mestre em Educação em } \\
\text { Ciências }\end{array}$ & 2013 & 2013 \\
\hline 9 & $\begin{array}{l}\text { Licenciada em Matemática, mestre em Engenharia } \\
\text { Oceânica, doutora em Educação em Ciências }\end{array}$ & 1997 & 2007 \\
\hline 10 & $\begin{array}{l}\text { Licenciada em Matemática, especialista em Educação e } \\
\text { Tecnologias da Informação, especialista em Matemática, } \\
\text { mestre e doutora em Educação Ambiental }\end{array}$ & 2001 & 2004 \\
\hline 11 & $\begin{array}{l}\text { Licenciada em Educação Artística, especialista em } \\
\text { Aplicações para a Web, mestre em Enfermagem, doutora }\end{array}$ & 2000 & 2011 \\
\hline
\end{tabular}


RELACult - Revista Latino-Americana de Estudos em Cultura e Sociedade

\begin{tabular}{|c|r|r|c|}
\hline & em Educação Ambiental & & \\
\hline 12 & Licenciada em Letras Português - Espanhol, mestrado em \\
& Educação Ambiental & 2000 & 2009 \\
& & & \\
\hline
\end{tabular}

Fonte: Os autores (2018)

O procedimento metodológico da pesquisa foi fundamentado na objetividade entre parênteses, na qual não existe uma forma de investigar independente do observador para validar o explicar, pois este está imerso na explicação. O observador acontece no observar, e como seres humanos, encontra-se na situação de observador quando começa a observar o que faz com o propósito de descrevê-lo e explicá-lo (MATURANA, 2009). Por isso, o explicar dessa pesquisa tem relação com a maneira como se pergunta e opera o fenômeno investigado: Como são operadas as tecnologias digitais pelos professores de Matemática no Ensino Superior?

Para atender esse questionamento foi utilizado um circuito de quatro fóruns que problematizavam ações e situações educativas com as tecnologias digitais. O grupo foi dividido em quatro subgrupos e estes interagiram com um fórum a partir da problematização inicial. Na sequência, foi solicitada a alternância dos subgrupos nos fóruns e estes passavam a interagir não somente com a problematização inicial, mas também com as respostas dos colegas. Essa alternância ocorreu até todos os subgrupos tivessem interagido nos quatro fóruns.

Para organizar e articular os registros gerados nos quatro fóruns e sua relação com o a questão de pesquisa, se fez uso do Discurso do Sujeito Coletivo (DSC) de Lefèvre e Lefèvre (2005), por ser uma forma de organizar os discursos pela análise de diferentes materiais verbais que constituem seu corpus, possibilitando assim o exercício de produzir e expressar sentidos no que se refere o operar da tecnologia digital para ensinar Matemática na Educação Superior. Os autores apontam quatro operações para construir o discurso e cada uma delas influencia na compreensão e na constituição do discurso coletivizado.

As Expressões-Chave (E-Ch) é a primeira operação e se constituem de fragmentos contínuos ou descontínuos dos discursos, selecionados pelo pesquisador e que manifestam a essência do conteúdo do depoimento. Nesta pesquisa, as E-Ch foram geradas das problematizações do circuito dos fóruns realizado com os integrantes do Grupo EaD-TEC.

A segunda operação do DSC é definida como Ideias Centrais (IC), as quais apontam de maneira sintética os sentidos das E-Ch. As IC são abstratas e tem como objetivo identificar cada sentido ou posicionamento presente nos depoimentos. Ao se analisar as inúmeras E-Ch 
emergiram as IC as quais foram denominadas: finalidade conceitual; finalidade pedagógica; prática docente; formação profissional; falta de formação; formação permanente; conhecimento sobre as tecnologias; acesso à tecnologia; e presença das tecnologias.

A ancoragem (AC) é a terceira operação, que é a expressão de uma determinada teoria ou marca ideológica que o depoente manifesta. Para Lefèvre e Lefèvre (2012), pode-se considerar a AC como afirmações genéricas utilizadas pelos sujeitos para enquadrar situações particulares. Além disso, para que haja AC é necessário encontrar no depoimento, expressões discursivas explícitas a respeito dela. No que se refere aos registros dos quatro fóruns, percebeu-se o surgimento de seis ancoragens intituladas: dar-se conta nas práticas pedagógicas; cultura digital; enação; interação; formação docente; e aprender.

A partir destas operações que compõem a técnica do DSC foi gerado o discurso coletivo denominado "O aprender matemática enatuado na docência pelas tecnologias digitais" e que será analisado na próxima seção com a intenção de explicar o fenômeno investigado.

\section{Compreendendo o discurso coletivizado}

O discurso "O aprender matemática enatuado na docência pelas tecnologias digitais" evidencia que diferentes experiências de ação e reflexão sobre o operar das tecnologias digitais instigam o estudante a experimentar, fazer e interagir, o que permite significar e aprender a Matemática em uma cultura digital.

De acordo com Maturana e Verden-Zöller (2004), a cultura é um modo de convivência determinada por uma rede de conversação que é vivida na recorrência das ações e emoções dos sujeitos. Assim, pode-se explicar a cultura pela transformação de seus dispositivos de produção e de construção do conhecimento, bem como pensar a cultura digital como produções e criações a partir do operar das tecnologias digitais.

A cultura digital pode ser compreendida não apenas como o uso de ferramentas digitais, mas também, como processos, experiências, vivências e escolhas que ocorrem frente ao excesso de informações e serviços que circulam pelos bancos de dados, redes e dispositivos digitais. Lévy (2000, p. 17) denomina a cultura digital como sendo a cibercultura, e a define como um "conjunto de técnicas, de práticas, de atitudes, de modos de pensamento e de valores que se desenvolvem juntamente com o crescimento do ciberespaço". Dessa forma, as influências que a tecnologia digital tem sobre o cotidiano das pessoas e, especificamente, nos processos educacionais provocam outras mediações entre a abordagem do professor, a compreensão do estudante e o conteúdo problematizado, pois a maneira como estes sujeitos 
operam as tecnologias digitais no ambiente educativo podem modificar o comportamento deles e alterar a lógica da sala de aula.

Os docentes vêm adotando uma prática pedagógica que insere o operar das tecnologias digitais de forma rica, recursiva, reflexiva e relacional na formação de profissionais (GOULART, 2010). Porém, o que fará com que os profissionais, ou futuros profissionais estejam preparados e atualizados para usar os artefatos tecnológicos na sua ação são os interesses, os desejos e as necessidades coligados.

No discurso coletivo, é apontado pelo sujeito de pesquisa, que a tecnologia digital é usada "como objeto de exploração técnica e pedagógica para o meu planejamento, especialmente para pesquisas e busca de situações problemas para aproximar os conceitos matemáticos do que é desejado que seja fortalecido, reforçado na mente do indivíduo. Costumo usar programas de representação geométrica $3 D$ por exemplo, para que realizem trabalhos de pesquisa em grupo, vídeos elaborados pelos alunos elou professor e AVA" (extrato do DSC). Essa concepção do professor se refere à inserção das tecnologias digitais em sua prática pedagógica para a compreensão conceitual da Matemática. Assim, acredita-se que utilizar a tecnologia com essa finalidade pode desencadear o interesse do estudante, pois a complexidade de alguns processos pode ser visibilizados, bem como simulados pelas tecnologias, auxiliando na compreensão dos conceitos matemáticos.

Ao operar a tecnologia digital, o sujeito está em interação com esta, o que faz emergir outros significados que influenciam suas ações em uma determinada situação. O fazer emergir na ação é definida por Varela (1997) como a enação, que apresenta como princípio lógico a recursividade, que extrapola a linearidade, o que garante dinâmicas de interação em que são incluídos os sentimentos, as emoções, a dimensão história e o contexto em que ocorrem os fenômenos, entre os sujeitos e destes com o meio.

As tecnologias digitais possibilitam a gravação dos passos na construção de um objeto matemático ou na resolução de um problema, a captura e reprodução de procedimentos realizados, que geram um reolhar e, portanto, uma reflexão sobre um processo. Um exemplo desta situação, pode ser observado no excerto do discurso coletivo que aponta "no curso de licenciatura em matemática os alunos 'se dão conta' do quanto trabalhar a variação de parâmetros de uma função se torna mais interessante aportado pelo uso das tecnologias, por exemplo, um problema de otimização, minimizar uma função custo". Operar softwares dinâmicos gera diferentes possibilidades, que proporciona interatividade e interação entre os estudantes, a tecnologia digital e o conceito problematizado. 
Esse fato permite que os estudantes tenham a oportunidade de concentrar seus esforços na interpretação e na análise das situações que envolvem o problema, assim como simular diferentes condições para enriquecer a sua análise. Dessa forma, Bona (2009, p. 36) nos diz que com uma proposta educativa estruturada e com finalidade pedagógica permite que o estudante dê novos significados as atividades de ensino e "os softwares educativos podem ser um notável auxiliar para o aluno adquirir conceitos em determinadas áreas do conhecimento". A final, utilizar os software durantes as práticas de ensino possibilita ao estudante construir seu conhecimento, por meio da visualização e análise de situações, conforme é evidenciado no DSC: "toda a parte matemática que resolve o problema é mostrada e eles [estudantes] dizem sentir evolução, quando precisam resolver problemas e pesquisar conteúdos para poder ter técnicas para atacar problemas". Para Menegais, Fagundes e Sauer (2014), quando o professor gera situações a partir da utilização de tecnologias digitais articuladas às práticas pedagógicas, considerando o contexto da cultura digital em que os estudantes estão inseridos e respeitando os seus ritmos e as características de cada um, a recorrência no operar dos artefatos tecnológicos para pensar e aprender podem originar mudanças pedagógicas.

No tocante aos processos pedagógicos, a adoção de artefatos tecnológicos conduz os sujeitos, através da interação e da interatividade, ao estabelecimento de outras condições de participação e de cooperação nas etapas do processo do aprender (LÉVY, 2010). Assim, as tecnologias digitais, como produto de interações do ser humano contribuem com os processos educativos.

Pablos (2006) indica que a tecnologia digital não somente representa uma oferta pedagógica, como também um ambiente virtual de convívio social, com possibilidades de interações relacionadas a objetos de aprendizagem. Sua validade educativa se sustenta no operar das tecnologias digitais, ou seja, como os sujeitos se apropriam dessas ferramentas para desenvolver suas práticas pedagógicas.

No DSC observa-se o dar-se conta dos professores em relação à importância da utilização das tecnologias digitais para potencializar o ensino e a aprendizagem da Matemática, bem como para analisar situações-problemas e encontrar suas soluções, pois "acessando os aplicativos existentes e a forma como eles são utilizados, sempre buscando mostrar a parte matemática que foi implementada para chegar no resultado seriam mais prazerosos e exitosos quando desenvolvidas por tecnologias que não fossem somente através de fórmulas específicas". Ademais, a adoção de artefatos tecnológicos na atividade educativa pode desenvolver várias possibilidades aos estudantes como, por exemplo, a pesquisa individual ou em grupo, a comunicação e a interação entre eles para a execução das atividades 
em colaboração e para atingir seus objetivos (SCHWERTL e LEONEL, 2016). Em processos de colaboração, os sujeitos se apoiam e buscam estabelecer relações de confiança, os quais podem legitimar os diferentes saberes e respeitar os outros na convivência, o que corrobora para fundar ou manter distintas relações sociais em quaisquer ações conjuntas, algumas delas fundamentais e constitutivas do humano (MATURANA, 2014).

A intenção é que o espaço educativo seja para além do aprender, mas que possibilite conhecer e aceitar o outro como legítimo outro na sua existência, valorizando as diferenças e estimando a bagagem cultural de cada um. Segundo Primo (2008), nesse processo de colaboração e de legitimação do outro, os laços criados promovem a socialização de experiências, seja pela presencialidade, seja por meio das tecnologias digitais em rede, o que pode levar os estudantes a agirem com maior interesse durante a construção do conhecimento.

As tecnologias digitais podem auxiliar os estudantes de forma a gerar estratégias de aprendizagem, seja trocando informações a partir de grupos em redes sociais, no acesso à vídeo-aula ou durante a construção de elementos matemáticos em software, como é o caso explicitado no extrato do DSC referindo-se às aulas de Geometria que "seriam muito mais fáceis de serem desenvolvidas através das tecnologias, software como geogebra, cabrigeométrico, simuladores digitais para o ensino do traçado de curvas e superfícies, lousa digital, objetos virtuais de aprendizagem para visualização gráfica”. Especificamente na matemática, durante o ensino da Geometria, o estudante quando opera a tecnologia digital pode visualizar o passo a passo de sua solução por um aplicativo, desenhar uma curva ou plotar em um software, simulando diferentes comportamentos a partir da variação dos parâmetros de um problema.

Nessa perspectiva, Pretto e Assis (2008, p. 81), comentam que a universidade e a escola se tornam espaços "de produção, ampliação e multiplicação de culturas, apropriando-se das tecnologias". Por isso, as tecnologias digitais, quando presentes nas práticas de ensino, transformam os professores e estudantes em produtores de culturas e conhecimentos, superando a lógica de serem recursos somente de consumo da informação.

Cabe salientar que as tecnologias digitais são instrumentos que podem promover a integração entre os sujeitos por meio das possibilidades interativas que se estabelecem pelo meio digital. De acordo com Silva (2005), a interatividade é a comunicação entre os sujeitos, potencializada pelas tecnologias digitais; é a expressão da cultura, da participação e da colaboração que emerge na confluência complexa entre o sujeito e as tecnologias digitais em rede geradas pelo social. 
Esta forma interativa que é promovida pelo operar da tecnologia digital, pode dar significado ao ensino da Matemática, pois a maneira com que se age no ciberespaço se funde a construção do conhecimento. Da mesma forma, para Braga e Paula (2010), ao se voltar para o ensino, o processo de aprender está imbricado, um vez que há uma reorganização do pensamento por meio de uma ação que promove a reflexão, favorecido pelas interações que ocorrem no operar da tecnologia digital, e que gera novas configurações na construção do conhecimento. Esta perspectiva, também é encontrada pelos sujeitos da pesquisa, quando no discurso coletivizado é evidenciado que "o professor tem papel fundamental para estimular a reflexão dentro do contexto proposto em sala de aula e proporcionar oportunidade de interação".

Por isso, compreender que o processo de reflexão possui verdades relativas aos seres humanos, tem relação com a forma com que se vive e se age no mundo por meio das experiências geradas. Isso implica dizer que as pessoas são influenciadas por fatores sociais e culturais, mas que esses também são, ao mesmo tempo, gerados pelo modo de pensar e agir, bem como pela maneira que cada um vive em sociedade.

Por esta razão, é importante a proposição de diferentes atividades em sala de aula, operando as tecnologias digitais pedagogicamente para promover distintas "experiências que contemplem a criatividade, autonomia, envolvimento de todos na prática pedagógica para direcionar os estudantes a locais de pesquisa e aplicações relativas às áreas de atuação do futuro profissional" (excerto do DSC). As experiências geradas por estas atividades permitem ao estudante, produzir conhecimentos, desenvolver estratégias para resolução de problemas e criatividade para solucioná-las (SCHEFFER, 2012). Assim, a incorporação recorrente das tecnologias digitais em sala de aula, em especial no ensino de matemática, pode resultar na criação de ambientes de aprendizagem que possibilitem ao estudante o desenvolvimento de novos conceitos e a consolidação da aprendizagem.

\section{Conclusões}

Pela pesquisa realizada, compreende-se que questões vinculadas ao operar das tecnologias digitais no ato de ensinar e de aprender adquirem mutabilidade que determinam as dinâmicas de trabalho, marcadas por processos de contínua aquisição de informação e/ou construção de conhecimentos. Ademais, a incorporação das tecnologias digitais viabilizando a expressão, o compartilhamento e a produção de ações, emoções e experiências em um coordenar recorrente e recursivo poderá viabilizar uma proposta de formação no espaço universitário que ultrapasse a sala de aula, que potencialize o acesso e a convivência em uma 
cultura digital trazendo desafios à prática pedagógica. Assim, acredita-se que se a formação dos professores universitários estiver ancorada em uma perspectiva que opera a tecnologia digital como uma forma de agir, viver e compreender esse operar no seu fazer profissional, esses poderão significar os artefatos tecnológicos nas atividades de cada profissão, o que transformará a cultura digital no ambiente educativo da Universidade.

Percebe-se que a cultura digital é gerada pelo uso recorrente da tecnologia a partir dos diferentes domínios do sujeito - interações afetivas, práticas cotidianas e atividades profissionais - que passa a ser incorporada por diferentes grupos sociais, dinamizando mudanças significativas nos modos de vida das mais diferentes sociedades. Verificou-se ainda que a recorrência do discurso analisado estava voltada ao desenvolvimento das práticas pedagógicas dos professores com o uso da tecnologia digital, em que estes se dão conta da possibilidade operativa desses recursos para a prática relacional dos conceitos matemáticos. Todavia, se aposta na ampliação da ação educativa para além de uma prática voltada aos conceitos, como uma prática relacional entre os sujeitos, como um conviver e compartilhar de uma experiência que produz sentido, conhecimentos e formas de viver.

Especificamente na formação de professores de Matemática, pensar para além dos conhecimentos da Matemática que irá ensinar, significa compreender a importância do desafio inerente ao processo de ensinar e de aprender e dos princípios em relação ao caráter ético de sua atividade docente. Por isso, propor e vivenciar projetos e ações que primam pelo trabalho coletivo entre os docentes e estudantes, bem como promover a experienciação de atividades com as tecnologias digitais pode ser uma forma de potencializar uma cultura de ensino que seja flexível, solidária e democrática frente à realidade multifacetada da sociedade em rede, superando e rompendo com a tendência fragmentada e desarticulada dos processos formativos atuais.

Assim, finda-se este trabalho apontando uma resposta a questão de pesquisa: como são operadas as tecnologias digitais pelos professores de Matemática no Ensino Superior? Tal problematização faz com que se anuncie que o operar da tecnologia digital na sala de aula promove processos de interação que estabelecem uma cultura digital entre os sujeitos que compõem o espaço educativo, que transformam o meio e são transformados por este, através de fluxos recursivos em um modo humano de viver que envolve ações e emoções no espaço de sala de aula. 


\section{Referências}

BONA, B. O. Análise de softwares educativos para o ensino de Matemática nos anos inicias do ensino fundamental. Experiências em Ensino de Ciências, v. 4, n. 1, p. 35-55, 2009. ISSN 1982-2413. Disponível em: http://www.if.ufrgs.br/eenci/artigos/Artigo_ID71/v4_n1_a2009.pdf Acesso em: 18 jan. 2018.

BRAGA, M.; PAULA, R. M. O ensino de Matemática mediado pelas tecnologias da informação e comunicação - uma caracterização do elemento visualização segundo uma concepção fenomenológica. Revista Tecnologias na Educação, v. 2, n. 1, p. 1-19, 2010. ISSN 1984-4751. Disponível em: http://tecedu.pro.br/wp-content/uploads/2015/07/Art3vol12-julho-2010.pdf Acesso em: 24 fev. 2018.

GOULART, M. B. O uso do computador na formação inicial de professores de Matemática: integração de propostas curriculares em universidades públicas. In: BURAK, D.; PACHECO, E. R.; KLÜBER, T. E. (Orgs.). Educação Matemática: reflexões e ações. Curitiba: Editora CRV, 2010. p. 251-270.

LEFÈVRE, F.; LEFÈVRE, A. M. C. Pesquisa de representação social: um enfoque qualiquantitativo a metodologia do Discurso do Sujeito Coletivo. Brasília: Liber Livro Editora, 2012. 224 p.

LEFÈVRE, F.; LEFÈVRE, A. M. C. O discurso do sujeito coletivo: um novo enfoque em pesquisa qualitativa (desdobramentos). Caxias do Sul: Educs, 2005. 256 p.

LÉVY, P. Cibercultura. São Paulo: Editora 34, 2010. 272 p.

LÉVY, P. As tecnologias da inteligência: o futuro do pensamento na era da informática. São Paulo: Editora 34, 2000. 208 p.

MATURANA, H. Cognição, ciência e vida cotidiana. Belo Horizonte: Editora UFMG, 2014. 203 p.

MATURANA, H. Emoções e linguagem na educação e na política. Belo Horizonte: Editora UFMG, 2009. 98 p.

MATURANA, H.; VERDEN-ZÖLLER, G. Amar e brincar: fundamentos esquecidos do humano do patriarcado à democracia. São Paulo: Palas Athena, 2004. 264 p.

MENEGAIS, D. A. F. N.; FAGUNDES, L. C.; SAUER, L. Z. Impacto da inserção de tecnologias digitais na formação inicial de professores de matemática egressos de uma Universidade Pública Federal. Revista Novas Tecnologias na Educação, v. 12, n. 2, p. 1-9, 2014. ISSN 1679-1916. Disponível em: https://seer.ufrgs.br/renote/article/view/53560 Acesso em: 23 fev. 2018.

PABLOS, J. A visão disciplinar no espaço das tecnologias da informação e comunicação. In: SANCHO, J. M. HERNÁNDEZ, F. (Orgs.). Tecnologias para transformar a educação. Porto Alegre: Artmed, 2006. p. 63-83. 
PRETTO, N. L. O desafio de educar na era digital: educações. Revista Portuguesa de Educação, v. 24, n. 1, p. 95-118, 2011. ISSN: 0871-9187. Disponível em: https://revistas.rcaap.pt/rpe/article/view/3042/2459. Acesso em: 02 dez. 2018.

PRETTO, N. L.; ASSIS, A. Cultura digital e educação: redes já! In: PRETTO, N. L.; SILVEIRA, S. A. (Orgs.). Além das redes de colaboração: Internet, diversidade cultural e tecnologias do poder. Salvador: EDUFBA, 2008. p. 75-84.

PRIMO, A. O aspecto relacional das interações na Web 2.0. In: AUTOUN, H. (Org.). Web 2.0: participação e vigilância na era da comunicação distribuída. Rio de Janeiro: Mouadx, 2008. p. 10-25.

SCHEFFER, N. F. A argumentação em Matemática na interação com tecnologias. Ciência e Natura, v. 34, n. 1, p. 23-38, 2012. ISSN 2179-460X. Disponível em: https://periodicos.ufsm.br/cienciaenatura/article/viewFile/9352/5503 Acesso em: 17 jan. 2018.

SCHWERTL, S. L.; LEONEL, A. A. Discussão de tópicos de Matemática Básica nos espaços sociais da Web 2.0: desafios e possibilidades a partir da análise de uma intervenção pedagógica. Revista Brasileira de Ensino de Ciência e Tecnologia, v. 9, n. 3, p. 83-102, 2016. ISSN: 1982-873X. Disponível em: https://periodicos.utfpr.edu.br/rbect/article/view/4434/pdf Acesso em: 23 fev. 2018.

SILVA, M. Interação e interatividade: sugestões para docência na cibercultura. In: PORTO, C. et al. (Orgs.). Pesquisa e mobilidade na cibercultura: itinerâncias docentes. Salvador: Edufba, 2015. p. 43-64.

VARELA, F. Prefácio de Francisco J. Varela à segunda edição. In: MATURANA, H.; VARELA, F. De máquinas e seres vivos: autopoiese - a organização do vivo. Porto Alegre: Artes Médicas, 1997. p. 34-61. 